\title{
Aspectos genéticos nas afecções do ombro*
}

\section{Genetic Aspects in Shoulder Disorders}

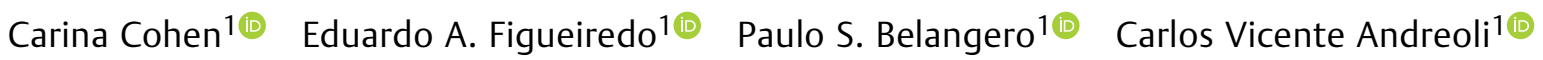 \\ Mariana Ferreira Leal ${ }^{2}$ Benno Ejnisman ${ }^{10}$
}
${ }^{1}$ Disciplina de Medicina do Esporte e Atividade Física , Centro de Traumatologia do Esporte, Universidade Federal de São Paulo, São Paulo, SP, Brasil
2 Departamento de Morfologia e Genética, Universidade Federal de São Paulo, São Paulo, SP, Brasil

\author{
Endereço para correspondência Carina Cohen, PhD, Avenida Lineu de \\ Paula Machado, 660, Jd Everest, São Paulo, SP, 05601-000, Brasil \\ (e-mail: cacohen18@gmail.com).
}

Rev Bras Ortop 2020;55(5):537-542.

\section{Resumo \\ Palavras-chave \\ - ombro \\ - polimorfismo genético \\ - expressão gênica}

A influência da herança genética tem sido cada vez mais investigada nas afecções do ombro, como a lesão do manguito rotador, instabilidade e ombro congelado. Ainda que os achados iniciais sejam pouco esclarecedores, é necessário construir progressivamente um banco de marcadores genéticos para catalogar perfis genômicos que, mais adiante, poderão contribuir para a previsão do risco da doença, desenvolvimento de melhores ferramentas de diagnóstico e tratamento. O presente artigo busca atualizar o que há de evidências de estudos genéticos na literatura para essas doenças, desde análises de polimorfismos, expressão de genes candidatos em tecidos e estudos de associação genômica ampla (GWAS, na sigla em inglês). Porém, é necessário apontar que existe grande dificuldade na replicação e utilização dos achados, principalmente em razão da falta de poder estatístico, da alta taxa de resultados falso-positivos e da grande quantidade de variáveis envolvidas.

The influence of genetic inheritance has been increasingly investigated in shoulder disorders, such as rotator cuff injury, instability and frozen shoulder. Although the initial findings are enlightening, it is necessary to progressively build a database of genetic markers to catalog genomic profiles that, later, may contribute for predicting the risk of the disease, as well as to the development of better diagnostic and treatment tools. The present article seeks to update what is evidence of genetic studies in the literature for these diseases, from polymorphism analyses, expression of candidate genes in tissues and broad genomic association studies (GWAS). However, it is necessary to point out that there is great difficulty in replicating and using the findings, mainly due to the lack of statistical power, the high rate of false-positive results and the large number of variables involved.

\footnotetext{
Trabalho desenvolvido na Disciplina de Medicina do Esporte e Atividade Física, Centro de Traumatologia do Esporte (CETE), Universidade Federal de São Paulo (UNIFESP), São Paulo, SP, Brasil.
}

recebido

05 de Setembro de 2019 aceito

12 de Novembro de 2019
DOI https://doi.org/

10.1055/s-0040-1702955. ISSN 0102-3616.
Copyright $\odot 2020$ by Sociedade Brasileira License terms de Ortopedia e Traumatologia. Published by Thieme Revinter Publicações Ltda, Rio de Janeiro, Brazil 


\section{Introdução}

A influência da genética tem sido cada vez mais investigada nas afecções do ombro, como a lesão do manguito rotador (LMR), instabilidade e ombro congelado (OC). Por se tratarem de doenças complexas ou multifatoriais, essas lesões são determinadas pela interação de fatores genéticos e ambientais. Uma característica das doenças complexas é que elas podem apresentar agregação familiar, pois é mais provável que os parentes de uma pessoa afetada compartilhem com ela mais alelos de predisposição à doença do que pessoas não aparentadas.

O genoma de quaisquer 2 indivíduos da espécie humana são idênticos em 99,9\% de sua sequência. ${ }^{1}$ Assim, a diferença entre indivíduos representa $0,1 \%$ do genoma. Dentre as variações, quando a frequência de uma variante alélica atinge mais do que $1 \%$ da população, essa variante passa a ser chamada "polimorfismo". O tipo mais frequentemente estudado são os polimorfismos de nucleotídeo único (SNP). Existem vários desenhos de estudos apropriados para a investigação genética das doenças multifatoriais, como os estudos de gêmeos, estudos de adoção, estudos de famílias, estudos de trios (propósito, mãe e pai) e estudos de casocontrole. $^{2}$

Para lesões do sistema musculoesquelético, grande parte dos estudos publicados investiga a frequência de polimorfismo de "genes candidatos" em casos e controles com base nas publicações da literatura. Os estudos caso-controle podem também ser realizados em larga escala, como os estudos de associação genômica ampla (GWAS, na sigla em inglês), que utilizam técnicas robustas de sequenciamento ou microarray (chips de oligonucleotídeos) para fazer uma varredura das variantes genéticas (geralmente centenas de milhares de SNPs) em um genoma humano. Os estudos de associação genômica ampla são, essencialmente, uma abordagem livre de hipótese, porque não faz suposições sobre a localização ou o significado funcional de locos associados ou seus produtos. ${ }^{2}$ Convém ressaltar que, além dos estudos baseados nas análises de DNA, os estudos de expressão gênica e proteica podem fornecer pistas de genes envolvidos, e o conhecimento da função biológica desses genes pode auxiliar na compreensão da fisiopatologia molecular da doença.

A maior parte dos estudos que avaliaram se variantes genéticas estão associadas ao risco de lesões do ombro investigou polimorfismos em genes que codificam proteínas presentes na matriz extracelular (MEC) ou que estão envolvidos direta ou indiretamente na homeostase e no processo de reparo dos tecidos envolvidos (ligamentos, tendões, e cápsula) cuja estrutura básica é a fibrila de colágeno. Variantes nos genes do colágeno podem alterar a estrutura primária do mesmo, gerando cadeias menos estáveis do que o normal, de forma afetar a estrutura capsular. As fibrilas dos tecidos articulares são compostas predominantemente pelo colágeno tipo I, que é responsável primariamente pela resistência fisiológica à tensão. Durante o processo de reparo de tendões e ligamentos, é postulado que colágeno III forma uma arquitetura primária que, posteriormente, é infiltrada e substituída por colágeno $\mathrm{I}^{3} \mathrm{O}$ colágeno tipo V é um regulador da fibrilogenese e se intercala nas fibras de colágeno tipo I, de forma que sua alteração pode gerar danos estruturais na cápsula, como foi sugerido em indivíduos com síndrome de Ehlers Danlos, cuja expressão do COL5 se mostrou diminuída em entre 25 e $30 \%$ dos pacientes com mutação desse gene. ${ }^{4}$

Adicionalmente, foram estudados outros colágenos, genes de moduladores da síntese de colágeno (TGFB, TGFB1), metaloproteinases, proteoglicanos que podem estar associados às fibrilas e glicoproteínas - por exemplo, fibronectina $(F N)$ e tenascina $C(T N C)$, que atuam na modulação do TGFB e no processo de reparo dos tendões por promover migração e adesão de fibroblastos às fibrilas. ${ }^{5,6}$ As citocinas, como o TGFB1 e seus receptores, podem desempenhar um papel fundamental nos processos inflamatórios e fibróticos, regulando várias proteínas da MEC, incluindo colágenos, FN1 e TNC. $^{7}$ É, sem dúvida, uma das citocinas mais intimamente envolvidas no processo de fibrose, e está presente em grandes quantidades em locais de inflamação crônica. ${ }^{8}$

As metaloproteinases da matriz (MMPs) são proteases dependentes do zinco responsáveis pela remodelação tecidual e pela degradação da MEC durante processos fisiológicos normais como proliferação celular, remodelação de tecidos, reprodução, diferenciação, angiogênese e apoptose, mas também participam em doenças como artrite, invasão tumoral, câncer e inflamação, podendo prejudicar a organização e o suporte estrutural dos tecidos. ${ }^{9}$ Essas enzimas são classificadas com base em sua preferência de substrato, incluindo colagenases (por exemplo, MMP1 e MMP13), estromelizinas (por exemplo, MMP3) e gelatinases (por exemplo, MMP2 e MMP9). As metaloproteinases da matriz são inibidas por uma classe de proteínas chamada TIMP.

\section{Lesão do Manguito Rotador}

Apesar das teorias de causas intrínsecas e extrínsecas para origem das lesões do manguito rotador (LMR), existem evidências preliminares da contribuição genética que levam à degeneração tendínea e à consequente ruptura dos tendões. ${ }^{10}$

Estudos da literatura demonstram que irmãos de indivíduos com LMR apresentam maior propensão a desenvolver lesão completa e risco de serem sintomáticos. ${ }^{11-14}$ Recentemente, em um estudo com 33 pares de gêmeos idosos (17 monozigóticos e 16 dizigóticos) Gumina et al. ${ }^{15}$ calcularam o índice de herdabilidade de $18 \%$, e a contribuição de $44 \%$ para o ambiente compartilhado e de $38 \%$ para o ambiente único. Estudos de expressão gênica demonstram diferentes comportamentos dos genes em relação à lesão do manguito rotador. Riley et al. ${ }^{16}$ avaliaram 10 pacientes e 24 controles e encontraram diminuição da expressão dos genes MMP2, MMP9, MMP13.

Lo et al. ${ }^{17,18}$ em estudo com tecido de 10 pacientes e 6 cadáveres, encontraram o aumento da expressão de MMP13 e inibição da expressão de MMP3, TIMP2, TIMP3 e TIMP4. Em 2005, o mesmo autor encontrou aumento da expressão de MMP13, COL1A1, COL3A1 e agrecano, além da inibição da expressão de decorina. 
Shindle et al. ${ }^{19}$ encontraram aumento da expressão de MMP9, MMP13, COX2 e COL1A1 bem como a diminuição da expressão de iNOS, VEGF, COL3A1 e biglican. Shirachi et al. ${ }^{20}$ analisaram a expressão de COL1A1 e COL3A1 na borda da lesão do tendão do supraespinal em 12 pacientes, tendo como controle cinco cadáveres frescos. Os autores correlacionaram a expressão desses genes com a integridade do reparo após o período de um ano de pós-operatório. Além disso, a expressão de COL1A1 foi associada com o tempo de início dos sintomas, sugerindo que o tratamento conservador não deva ser prolongado se os pacientes não apresentarem melhora após determinado período.

Robertson et al. ${ }^{21}$ avaliaram a expressão gênica de citocinas pró-inflamatórias, genes de remodelação tecidual e fatores de angiogênese em 35 pacientes com LMR completa. Houve correlação do aumento da expressão de MMP1 e MMP9 e falha da cicatrização do reparo. Gotoh et al. ${ }^{22}$ realizaram estudo com 24 pacientes e encontraram aumento da expressão de MMP3 e TIMP1 nos pacientes que tiveram reruptura após um ano do reparo da LMR. A expressão dos genes de colágeno não apresentou relação.

O primeiro estudo genético realizado por autores nacionais observou correlação entre a LMR e polimorfismos nos genes DEFB1, ESRRB, FGF3, FGF10 e FGFR1. Os autores também concluíram que sexo feminino e cor branca são os principais preditores deste tipo de lesão. ${ }^{23}$ Confirmando estes achados, mutações do gene ESRRB foram correlacionados à lesão em um estudo com 175 pacientes com LMR comparados a um grupo controle de 3.293 indivíduos de um banco de dados genético de acesso aberto para consulta (Illumina iControls database). ${ }^{24}$ Vale ressaltar que esta série de casos não distinguiu pacientes com lesões traumáticas e degenerativas.

Tashjian et al. ${ }^{25}$ utilizaram informação proveniente de um banco de dados genético populacional contendo uma amostra de 311 casos, compararam-na com 2641 indivíduos saudáveis e encontraram relação entre mutações nos genes SAP30BP e SASH1 em LMR. Ambos os genes apresentam relação direta com mecanismo de apoptose.

Em outra publicação realizada em população brasileira, foram avaliados 64 pacientes com LMR e foi apontada a associação com polimorfismos genéticos de metaloproteinases $M M P-1$ e $M M P-3 .^{26}$

Sejersen et al. ${ }^{27}$ em revisão sistemática, identificaram 2.199 estudos de análise de proteínas em tendinopatias e verificaram uma tendência de aumento da expressão de COL1, COL3, MMP1, MMP9, MMP13, TIMP1, VEGF e diminuição de MMP3. Chung et al. ${ }^{28}$ encontraram o aumento da expressão dos genes MMP9 e IL6 em diabéticos. Os autores sugeriram que essa diferença pode ser uma das explicações para o aumento da falha do reparo em pacientes diabéticos.

Nosso grupo, em 2017, descreveu por meio de estudo de Reação em cadeia da polimerase quantitativo em tempo real (qRT-qPCR), com amostra normalizada por meio dos genes HPRT1, TBP e $A C T B$, a diminuição da expressão de MMP1, MMP9 e MMP13 e o aumento de TIMP3 em indivíduos portadores de lesão em relação a controles. ${ }^{29} \mathrm{Em}$ outro estudo, a presença de polimorfirmos relacionados aos genes MMP-1, MMP-2, MMP-3, MMP-9, MMP-13, TIMP-1, TNC e COL5A1 em pacientes com LMR também foi observada. Estes autores encontraram 15 SNPs do gene TNC e os mesmos estavam significativamente associados a lesões degenerativas. ${ }^{30}$

Ahn et al. ${ }^{31}$ avaliaram 14 pacientes que foram submetidos ao reparo e concluíram que a regulação negativa de genes de resposta inflamatória e a regulação positiva de genes de diferenciação celular no momento da cirurgia estão relacionados à cicatrização do manguito rotador.

Treviño et al. ${ }^{32}$ avaliaram a expressão de proteases (catepsinas e $M M P$ ) de tecidos do tendão e músculo do supraespinal, além da cartilagem umeral de 30 ratos após LMR em 3 momentos distintos: 1, 3 e 12 semanas. Os autores concluíram que há um aumento significativo das proteases nos três tecidos, cada um com diferentes perfis, havendo inicialmente o aumento da expressão nos tendões e posteriormente na cartilagem umeral.

Lee et al. ${ }^{33}$ em estudo com ratos, analisaram 39.429 genes e acompanharam as alterações na expressão dos mesmos após 1 e 4 semanas de lesão e identificaram que a ruptura do manguito rotador induz a expressão de genes específicos relacionados ao envelhecimento, apoptose, atrofia e transporte de ácidos graxos. Os autores associaram que muitos genes que estão alterados possam ter um papel no processo de degeneração do tendão após a lesão. Em 2018, nosso grupo descreveu que a expressão alterada dos genes COL1A1, COL1A2, COL3A1, COL5A1, FN1, TNC, TGFB1, e TGFBR1 está envolvida no processo de degeneração nas rupturas do manguito rotador. ${ }^{34}$

Em estudo de revisão sistemática, Dabija et al. concluíram que apesar dos estudos anteriores fornecerem evidências preliminares de predisposição genética e familiar das LMR, há uma falta de grandes estudos genômicos que possam fornecer informações mais definitivas e orientar a detecção precoce de indivíduos em risco, reabilitação profilática e potenciais terapias gênicas e intervenções em medicina regenerativa. ${ }^{10}$

Um estudo mais recente realizou análise ampla usando perfil de expressão gênica GSE93661 do banco de dados Gene Expression Omnibus (GEO) e análises de bioinformática para investigar genes diferencialmente expressos (DEGs) em células satélites entre amostras de casos de lesão do supraespinal e controles do tendão subescapular. No total, 551 DEGs foram identificados, incluindo 272 DEGs hiper-regulados e 279 DEGs hiporregulados apontando uma série de genes (GNG13, GCG, NOTCH1, BCL2, NMUR2, PMCH, FFAR1, AVPR2, GNA14 e KALRN) e fornecendo assim pistas para especular que a via de sinalização GNG13/cálcio é altamente correlacionada com a atrofia por denervação no processo patológico das LMR. ${ }^{35}$

Há uma linha de pesquisa de nosso grupo em que analisamos a diferença de expressão gênica entre lesões parciais bursais e articulares, utilizando sistema de ontologia genética e a plataforma Sequenciamento de Nova Geração (NGS). A lesão parcial bursal mostrou-se geneticamente mais complexa por apresentar maior número de genes identificados pelo NGS, sendo que a maioria dos genes que apresentaram 
aumento de expressão estavam associados a fusão , adesão ou interação da célula com a matriz extracelular, enquanto na face articular foram identificados hiperexpressão do gene EGLN3, um sensor da saturação do oxigênio tecidual e supressão do gene ID1, um importante regulador de processos biológicos incluindo crescimento celular, senescência, diferenciação, apoptose, angiogênese e transformação neoplásica. (Tese de mestrado profissional de André Godinho, dados não publicados).

Por fim, em uma outra linha de investigação, que busca compreender se a infiltração gordurosa (IG) e inflamação retardam a cicatrização nas LMR, Thankam et al. avaliaram miRNAs de pacientes com LMR com e sem IG e inflamação e detectaram 13 miRNAs e 216 genes-alvos que interconectam o ponto de verificação metabólico 5' monofosfato-adenosina proteína quinase ativada (AMP-Q) e a via da molécula inflamatória TREM-1. ${ }^{36}$

\section{Instabilidade do ombro}

A instabilidade de ombro, como outras afecções ortopédicas, possui um possível componente genético. Foëx reportou a presença de deslocamentos recorrentes de ombro em três gerações de uma família do Reino Unido. ${ }^{37}$ Imazato $^{38}$ demonstrou que em pacientes com instabilidade multidirecional do ombro, as fibras colágenas da cápsula, músculos e pele são relativamente imaturas, mais solúveis e com menos crosslink que controles.

Em uma população da Suécia, foi observado que os homozigotos para o alelo raro no polimorfismo rs1800012 (no sítio de ligação $\mathrm{Sp} 1$ ) do gene que codifica a cadeia $\alpha 1$ do colágeno tipo 1 (COL1A1) era um fator protetor para instabilidade de ombro $(\mathrm{N}=126) .{ }^{39}$ Collins et al. ${ }^{40}$ reunindo os resultados de indivíduos caucasoides da Suécia e da África do Sul, investigaram se esse polimorfismo de COL1A1 estava associado ao risco de lesão do ligamento cruzado anterior do joelho, de instabilidade de ombro e de rupturas do tendão de Aquiles. Os autores descreveram que o genótipo TT era um fator protetor contra a lesão, quando todas as lesões foram combinadas e comparadas com indivíduos controles.

Inicialmente, Belangero et al. ${ }^{41}$ investigaram a expressão dos genes COL1A1, COL1A2, COL3A1 e COL5A1 na região anteroinferior (macroscopicamente alterada) e compararam com a região anterosuperior da cápsula glenoumeral de 18 pacientes com instabilidade anterior traumática do ombro (IATO). Identificaram expressão reduzida de COL5A1 na região superior. Os mesmos autores demonstraram em 2014 que expressão de COL1A1 e a razão COL1A1/COL1A2 estavam aumentadas em todas as regiões (anterossuperior, anteroinferior e posterior) da cápsula em pacientes com IATO quando comparados aos controles, e que essa razão parece reduzir na região anteroinferior quanto maior o tempo de sintomas. A razão entre COL1A1/COL5A1 também estava aumentada na região anteroinferior e posterior da cápsula. ${ }^{42}$ Em 2016, avaliaram genes relacionados ao processo de crosslink do colágeno e sugeriram que alterações de expressão nos genes do TGFB1, TGFBR1, LOX e PLOD2 podem desempenhar um papel na instabilidade do ombro. ${ }^{43}$ Por fim, ao avaliarem a expressão de genes que codificam proteínas da matriz extracelular (COMP, FN1, TNC e TNXB) encontraram maior expressão de TNC e FN1 na parte anteroinferior da cápsula, estando a FN1 diretamente correlacionada com a duração dos sintomas e com deslocamentos recorrentes em relação aos controles. ${ }^{44}$ Já a expressão de COMP estava reduzida, podendo estar associada com a integridade da cápsula após luxação do ombro, particularmente na porção macroscopicamente afetada.

\section{Ombro Congelado}

O histórico familiar positivo de ombro congelado (OC) é descrito em 9,5 até $20 \%$ dos casos, ${ }^{45,46}$ a prevalência calculada em estudo de gemelares é de $11,6 \%$ e a estimativa de herdabilidade de $42 \%{ }^{47} \mathrm{O}$ aspecto idiopático da lesão e estudos como o que relata o caso de irmãos monozigóticos com ombro congelado bilateral desenvolvido ao mesmo tempo $^{48}$ favorecem a teoria da propensão genética dos indivíduos acometidos. Além disso, outros dados que reforçam a provável influência genética de padrões complexos (interação gene-ambiente) do OC é a curiosa associação com a doença de Dupuytren (DD) que é uma doença complexa, multifatorial com forte componente genético conhecido. ${ }^{49}$

Existe grande semelhança histológica entre os processos fibroproliferativos das duas doenças, ${ }^{50}$ e foi relatada a associação entre condições fibróticas (ombro congelado e doença de Dupuytren), rigidez articular e artroplastias totais. Condições fibróticas apresentaram herdabilidade de $28 \%$. Esses achados são sugestivos de uma influência genética em um processo comum de doença subjacente que afeta os tecidos conjuntivos. ${ }^{51}$ Foi demonstrado o aumento da expressão de TGFB e TGFBR1 e diminuição dos níveis de MMP2 no tecido capsular de ombros acometidos. ${ }^{52} \mathrm{Em}$ acordo, Bunker et al. ${ }^{53}$ também demonstraram diminuição da expressão de mRNA de MMP1 e MMP2, semelhante à doença de Dupuytren.

Nosso grupo detectou na cápsula de oito pacientes operados de OC, em comparação com indivíduos controles, que essa hiperregulação de TGFBR1 se mostrou diretamente relacionada à duração dos sintomas de OC, sugerindo que a sinalização de TGFB deve estar envolvida no desenvolvimento da doença. Além disso, foi demonstrado aumento da expressão de mRNA de FN1 e de mRNA de TNC nos fragmentos de cápsula acometidos que pode estar envolvido no processo de inflamação e migração dos fibroblastos. ${ }^{54}$ Lubis et al. ${ }^{52}$ investigaram níveis séricos de MMPs, TIMPs e TGFß1 em OC e indivíduos normais usando método de ensaio de imunoabsorção enzimática (ELISA, na sigla em inglês). Os níveis basais de MMP1 e MMP2 foram significativamente menores, enquanto os níveis TIMP1, TIMP2 e TGFß1 foram significativamente maiores no grupo OC, achados semelhantes aos distúrbios fibroproliferativos na DD. ${ }^{9}$ Essas deficiências na produção de MMP1 podem refletir uma capacidade alterada para a remodelação local do tecido. ${ }^{55}$

Kabbabe et al. ${ }^{56}$ utilizaram a técnica de reação em cadeia da polimerase (PCR, na sigla em inglês) quantitativa para mostrar 
níveis de expressão aumentados de MMP3 e seu papel como mediador fibrogênico no OC em comparação com grupo controle, achados que estão de acordo com Xu et al. ${ }^{57}$ que concluíram que o aumento da expressão da variante de MMP3 rs650108 estava significativamente associada à susceptibilidade de OC em uma população Han chinesa.

Em 2017, Chen et al. ${ }^{58}$ estudaram SNPs da IL-1 $\beta$, MMP3, TGF- 1 1, e GDF5 em uma pequena população chinesa e encontraram que o genótipo $\mathrm{CC}$ do polimorfismo rs1143627 da IL-1 $\beta$ foi associado com menor risco de OC comparado ao genótipo TT ( $p=0.022)$ e que a IL-1 $\beta$ sérica foi expressa em um nível significativamente mais alto quando comparado ao grupo controle $(p<0,001)$.

Além disso, nosso grupo analisou 18 polimorfismos em genes que codificam proteínas envolvidas na homeostase da matriz extracelular da cápsula e da via de sinalização de TGFß1. Foram selecionados genes que codificam colágenos (COL1A1, COL5A1), glicoproteínas (FN1, TNC) genes envolvidos na sinalização do fator de crescimento transformador $\beta 1$ e seu receptor (TGFB1, TGFBR1), metaloproteinases (MMP2, MMP3, MMP9, MMP13) e inibidor tecidual da MMP2 (TIMP2 rs2277598). Enquanto nos homens foi apontada associação de polimorfismos de TGFB1 e TGFBR1, nas mulheres as metaloproteinases MMP2 e MMP9 foram apontadas como fatores de risco para desenvolvimento de OC. Apenas a MMP13 esteve relacionada com ambos os sexos. ${ }^{59}$ A MMP13, metaloproteinase do tipo colagenase, cliva o principal componente estrutural da cartilagem, o colágeno tipo II, efetuando, assim, a perda irreversível da arquitetura e função da Matriz extracelular (MEC). A expressão da colagenase MMP13 se mostrou significativamente elevada, na amostra de tecido de nódulos dos pacientes operados para a doença de Dupuytren, onde ocorre elevado turnover do colágeno; entretanto, elevados níveis de TIMP1 bloqueando a ação da MMP13, na quebra do colágeno, foi apontado como possível responsável pelo processo de contratura e fibrose nesses indivíduos. ${ }^{60}$

\section{Perspectivas}

A era da genética individual tem crescido exponencialmente nos últimos anos. Junto ao crescente número de publicações, existe uma grande frustração devido a resultados conflitantes, à expectativa de aplicação clinica dos resultados e à falta de replicação dos achados, principalmente em razão do baixo poder estatístico e da alta taxa de falso-positivos. Além disso, as doenças são controladas pela somatória de expressão de vários genes, porém cada um deles com efeito pequeno. Ainda se faz necessário catalogar diferentes polimorfismos relacionados ao ombro, uma vez que o perfil genômico permitirá definir um banco de marcadores genéticos que poderão contribuir para a previsão do risco das doenças. Dessa forma, o conhecimento das bases moleculares poderá ajudar no desenvolvimento de melhores ferramentas de prevenção, diagnóstico e tratamento.

\section{Conflito de Interesses}

Os autores declaram não haver conflito de interesses.

\section{Referências}

1 Lander ES, Linton LM, Birren B, et al. Initial sequencing and analysis of the human genome. Nature 2001;409(6822):860921

2 Leal MF, Belangero SI. Variantes genéticas nas lesões do sistema musculoesquelético em atletas. In: Cohen M, Abdalla RJ. Lesões nos Esportes: Diagnóstico, Prevenção e Tratamento. $2^{\text {a }}$. ed. Rio de Janeiro: Revinter; 2015:5-56

3 Frank CB. Ligament structure, physiology and function. J Musculoskelet Neuronal Interact 2004;4(02):199-201

4 Mitchell AL, Schwarze U, Jennings JF, Byers PH. Molecular mechanisms of classical Ehlers-Danlos syndrome (EDS). Hum Mutat 2009;30(06):995-1002

5 Chiquet-Ehrismann R, Tucker RP. Tenascins and the importance of adhesion modulation. Cold Spring Harb Perspect Biol 2011;3(05): a004960

6 Dallas SL, Sivakumar P, Jones CJ, et al. Fibronectin regulates latent transforming growth factor-beta (TGF beta) by controlling matrix assembly of latent TGF beta-binding protein-1. J Biol Chem 2005; 280(19):18871-18880

7 Badalamenti MA, Sampson SP, Hurst LC, Dowd A, Miyasaka K. The role of TGF-beta in Dupuytren's disease. J Hand Surg Am 1996;21 (02):210-215

8 Wahl SM, Costa GL, Mizel DE, Allen JB, Skaleric U, Mangan DF. Role of transforming growth factor beta in the pathophysiology of chronic inflammation. J Periodontol 1993;64(5, Suppl) 450-455

9 Ulrich D, Hrynyschyn K, Pallua N. Matrix metalloproteinases and tissue inhibitors of metalloproteinases in sera and tissue of patients with Dupuytren's disease. Plast Reconstr Surg 2003; 112(05):1279-1286

10 Dabija DI, Gao C, Edwards TL, Kuhn JE, Jain NB. Genetic and familial predisposition to rotator cuff disease: a systematic review. J Shoulder Elbow Surg 2017;26(06):1103-1112

11 Harvie P, Ostlere SJ, Teh J, et al. Genetic influences in the aetiology of tears of the rotator cuff. Sibling risk of a full-thickness tear. J Bone Joint Surg Br 2004;86(05):696-700

12 Gwilym SE, Watkins B, Cooper CD, et al. Genetic influences in the progression of tears of the rotator cuff. J Bone Joint Surg Br 2009; 91(07):915-917

13 Tashjian RZ, Farnham JM, Albright FS, Teerlink CC, CannonAlbright LA. Evidence for an inherited predisposition contributing to the risk for rotator cuff disease. J Bone Joint Surg Am 2009;91 (05):1136-1142

14 Tashjian RZ, Saltzman EG, Granger EK, Hung M. Incidence of familial tendon dysfunction in patients with full-thickness rotator cuff tears. Open Access J Sports Med 2014;5:137-141

15 Gumina S, Villani C, Arceri V, et al. Rotator Cuff Degeneration: The Role of Genetics. J Bone Joint Surg Am 2019;101(07):600605

16 Riley GP, Curry V, DeGroot J, et al. Matrix metalloproteinase activities and their relationship with collagen remodelling in tendon pathology. Matrix Biol 2002;21(02):185-195

17 Lo IK, Boorman R, Marchuk L, Hollinshead R, Hart DA, Frank CB. Matrix molecule mRNA levels in the bursa and rotator cuff of patients with full-thickness rotator cuff tears. Arthroscopy 2005; 21(06):645-651

18 Lo IK, Marchuk LL, Hollinshead R, Hart DA, Frank CB. Matrix metalloproteinase and tissue inhibitor of matrix metalloproteinase mRNA levels are specifically altered in torn rotator cuff tendons. Am J Sports Med 2004;32(05):1223-1229

19 Shindle MK, Chen CC, Robertson C, et al. Full-thickness supraspinatus tears are associated with more synovial inflammation and tissue degeneration than partial-thickness tears. J Shoulder Elbow Surg 2011;20(06):917-927

20 Shirachi I, Gotoh M, Mitsui Y, et al. Collagen production at the edge of ruptured rotator cuff tendon is correlated with postoperative cuff integrity. Arthroscopy 2011;27(09):1173-1179 
21 Robertson CM, Chen CT, Shindle MK, Cordasco FA, Rodeo SA, Warren RF. Failed healing of rotator cuff repair correlates with altered collagenase and gelatinase in supraspinatus and subscapularis tendons. Am J Sports Med 2012;40(09):1993-2001

22 Gotoh M, Mitsui Y, Shibata H, et al. Increased matrix metalloprotease-3 gene expression in ruptured rotator cuff tendons is associated with postoperative tendon retear. Knee Surg Sports Traumatol Arthrosc 2013;21(08):1807-1812

23 Motta GdaR, Amaral MV, Rezende E, et al. Evidence of genetic variations associated with rotator cuff disease. J Shoulder Elbow Surg 2014;23(02):227-235

24 Teerlink CC, Cannon-Albright LA, Tashjian RZ. Significant association of full-thickness rotator cuff tears and estrogen-related receptor- $\beta$ (ESRRB). J Shoulder Elbow Surg 2015;24(02):e31-e35 Erratum in: J Shoulder Elbow Surg 2016;25(5):864

25 Tashjian RZ, Granger EK, Farnham JM, Cannon-Albright LA, Teerlink CC. Genome-wide association study for rotator cuff tears identifies two significant single-nucleotide polymorphisms. J Shoulder Elbow Surg 2016;25(02):174-179

26 Assunção JH, Godoy-Santos AL, Dos Santos MCLG, Malavolta EA, Gracitelli MEC, Ferreira Neto AA. Matrix Metalloproteases 1 and 3 Promoter Gene Polymorphism Is Associated With Rotator Cuff Tear. Clin Orthop Relat Res 2017;475(07):1904-1910

27 Sejersen MH, Frost P, Hansen TB, Deutch SR, Svendsen SW. Proteomics perspectives in rotator cuff research: a systematic review of gene expression and protein composition in human tendinopathy. PLoS One 2015;10(04):e0119974

28 Chung SW, Choi BM, Kim JY, et al. Altered Gene and Protein Expressions in Torn Rotator Cuff Tendon Tissues in Diabetic Patients. Arthroscopy 2017;33(03):518-526.e1

29 Leal MF, Caires Dos Santos L, Martins de Oliveira A, et al. Epigenetic regulation of metalloproteinases and their inhibitors in rotator cuff tears. PLoS One 2017;12(09):e0184141

30 Kluger R, Burgstaller J, Vogl C, Brem G, Skultety M, Mueller S. Candidate gene approach identifies six SNPs in tenascin-C (TNC) associated with degenerative rotator cuff tears. J Orthop Res 2017; 35(04):894-901

31 Ahn JO, Chung JY, Kim DH, Im W, Kim SH. Differences of RNA Expression in the Tendon According to Anatomic Outcomes in Rotator Cuff Repair. Am J Sports Med 2017;45(13):2995-3003

32 Treviño EA, McFaline-Figueroa J, Guldberg RE, Platt MO, Temenoff JS. Full-thickness rotator cuff tear in rat results in distinct temporal expression of multiple proteases in tendon, muscle, and cartilage. J Orthop Res 2019;37(02):490-502

33 Lee YS, Kim JY, Kim HN, Lee DW, Chung SW. Gene Expression Patterns Analysis in the Supraspinatus Muscle after a Rotator Cuff Tear in a Mouse Model. BioMed Res Int 2018;2018:5859013

34 Santoro Belangero P, Antônio Figueiredo E, Cohen C, et al. Changes in the expression of matrix extracellular genes and TGFB family members in rotator cuff tears. J Orthop Res 2018;36(09):2542-2553

35 Ren YM, Duan YH, Sun YB, Yang T, Tian MQ. Bioinformatics analysis of differentially expressed genes in rotator cuff tear patients using microarray data. J Orthop Surg Res 2018;13(01):284

36 Thankam FG, Boosani CS, Dilisio MF, Gross RM, Agrawal DK. Genes interconnecting AMPK and TREM-1 and associated microRNAs in rotator cuff tendon injury. Mol Cell Biochem 2019;454(1-2):97-109

37 Foëx BA. Three generations of recurrent dislocated shoulders. Emerg Med J 2001;18(02):148-149

38 Imazato Y. [Etiological considerations of the loose shoulder from a biochemical point of view-biochemical studies on collagen from deltoid and pectoral muscles and skin]. Nippon Seikeigeka Gakkai Zasshi 1992;66(10):1006-1015

39 Khoschnau S, Melhus H, Jacobson A, et al. Type I collagen alpha1 Sp1 polymorphism and the risk of cruciate ligament ruptures or shoulder dislocations. Am J Sports Med 2008;36(12):2432-2436

40 Collins M, Posthumus M, Schwellnus MP. The COL1A1 gene and acute soft tissue ruptures. Br J Sports Med 2010;44(14):1063-1064
41 Belangero PS, Leal MF, de Castro Pochini A, Andreoli CV, Ejnisman B, Cohen M. Profile of collagen gene expression in the glenohumeral capsule of patients with traumatic anterior instability of the shoulder. Rev Bras Ortop 2014;49(06):642-646

42 Belangero PS, Leal MF, Figueiredo EA, et al. Gene expression analysis in patients with traumatic anterior shoulder instability suggests deregulation of collagen genes. J Orthop Res 2014;32 (10):1311-1316

43 Belangero PS, Leal MF, Cohen C, et al. Expression analysis of genes involved in collagen cross-linking and its regulation in traumatic anterior shoulder instability. J Orthop Res 2016;34(03):510-517

44 Belangero PS, Leal MF, Figueiredo EA, et al. Differential expression of extracellular matrix genes in glenohumeral capsule of shoulder instability patients. Connect Tissue Res 2016;57(04):290-298

45 Cohen C, Ejnisman B. Epidemiology of frozen shoulder. In: Itoi E, Arce G, Bain GI, Diercks RL, Guttmann D, Imhoff AB, et al. Shoulder stiffness. Berlin: Springer Verlag; 2015:21-30

46 Hand GC, Athanasou NA, Matthews T, Carr AJ. The pathology of frozen shoulder. J Bone Joint Surg Br 2007;89(07):928-932

47 Hakim AJ, Cherkas LF, Spector TD, MacGregor AJ. Genetic associations between frozen shoulder and tennis elbow: a female twin study. Rheumatology (Oxford) 2003;42(06):739-742

48 Hirschhorn P, Schmidt JM. Frozen shoulder in identical twins. Joint Bone Spine 2000;67(01):75-76

49 Larsen S, Krogsgaard DG, Aagaard Larsen L, Iachina M, Skytthe A, Frederiksen H. Genetic and environmental influences in Dupuytren's disease: a study of 30,330 Danish twin pairs. J Hand Surg Eur Vol 2015;40(02):171-176

50 Bunker TD, Anthony PP. The pathology of frozen shoulder. A Dupuytren-like disease. J Bone Joint Surg Br 1995;77(05): 677-683

51 Williams FM, Kalson NS, Fabiane SM, Mann DA, Deehan DJ. Joint Stiffness Is Heritable and Associated with Fibrotic Conditions and Joint Replacement. PLoS One 2015;10(07):e0133629

52 Lubis AM, Lubis VK. Matrix metalloproteinase, tissue inhibitor of metalloproteinase and transforming growth factor-beta 1 in frozen shoulder, and their changes as response to intensive stretching and supervised neglect exercise. J Orthop Sci 2013; 18(04):519-527

53 Bunker TD, Reilly J, Baird KS, Hamblen DL. Expression of growth factors, cytokines and matrix metalloproteinases in frozen shoulder. J Bone Joint Surg Br 2000;82(05):768-773

54 Cohen C, Leal MF, Belangero PS, et al. The roles of Tenascin C and Fibronectin 1 in adhesive capsulitis: a pilot gene expression study. Clinics (São Paulo) 2016;71(06):325-331

55 Brown ID, Kelly IG, McInnes IB. Detection of matrix metalloproteinases in primary frozen shoulders. J Bone Joint Surg Br 2008;90 (Suppl 2):364

56 Kabbabe B, Ramkumar S, Richardson M. Cytogenetic analysis of the pathology of frozen shoulder. Int J Shoulder Surg 2010;4(03): 75-78

57 Xu Q Gai PY, Lv HL, Li GR, Liu XY. Association of MMP3 genotype with susceptibility to frozen shoulder: a case-control study in a Chinese Han population. Genet Mol Res 2016;15(01):. Doi: 10.4238/gmr.15017228

58 Chen W, Meng J, Qian H, et al. A Study of IL-1 $\beta$, MMP-3, TGF- $\beta 1$, and GDF5 Polymorphisms and Their Association with Primary Frozen Shoulder in a Chinese Han Population. BioMed Res Int 2017; 2017:3681645

59 Cohen C, Leal MF, Loyola LC, et al. Genetic variants involved in extracellular matrix homeostasis play a role in the susceptibility to frozen shoulder: A case-control study. J Orthop Res 2019;37 (04):948-956

60 Johnston P, Chojnowski AJ, Davidson RK, Riley GP, Donell ST, Clark IM. A complete expression profile of matrix-degrading metalloproteinases in Dupuytren's disease. J Hand Surg Am 2007;32(03): 343-351 